\title{
Vestibular vertigo is associated with abnormal sleep duration
}

\author{
Monirah Albathi $^{\mathrm{a}, \mathrm{b}, *}$ and Yuri Agrawal ${ }^{\mathrm{a}}$ \\ ${ }^{a}$ Department of Otolaryngology-Head and Neck Surgery, School of Medicine, School of Public Health, \\ The Johns Hopkins University, Baltimore, MD, USA \\ ${ }^{\mathrm{b}}$ Department of Surgery, College of Medicine, Princess Nourah University Riyadh, Riyadh, Saudi Arabia
}

Received 16 September 2016

Accepted 14 April 2017

\begin{abstract}
.
BACKGROUND/OBJECTIVE: Several small studies in animals and humans have suggested a relationship between vestibular function and sleep. In this study, we evaluate the association between vestibular vertigo and sleep duration in a large, representative sample of US adults.

METHODS: We used data from the National Health Interview Survey, which administered a Balance Supplement in 2008 in a sample of 20,950 adult respondents. We evaluated the cross-sectional association between vestibular vertigo (based on a well-validated definition) and sleep duration (defined as short $<6$ hours, normal $6-8$ hours, and long $>8$ hours). We performed multiple and multinomial logistic regression analyses to estimate the odds ratio and relative risk ratio (RRR) of impaired sleep duration compared to normal sleep duration associated with vestibular vertigo. Analyses were adjusted for demographic, lifestyle and health behavior characteristics as well as relevant comorbid conditions.

RESULTS: Thirty percent of individuals with vestibular vertigo reported abnormal sleep duration (15.5\% short duration and $14.8 \%$ long duration). In adjusted analyses, individuals with vestibular vertigo had a 1.75 (95\% CI 1.45-2.11) RRR of having short sleep duration compared to individuals without vestibular vertigo, and a 1.55 (95\% CI 1.26-1.91) RRR of having long sleep duration compared to individuals without vestibular vertigo.

CONCLUSION: This study presents epidemiologic evidence to support the association between vestibular function and sleep duration. Individuals with vestibular vertigo had a higher RRR for abnormally short or long sleep duration. Further work is needed to evaluate the causal direction(s) of this association.
\end{abstract}

Keywords: Vestibular vertigo, vestibular system, sleep duration, National Health Interview Survey

\section{Introduction}

The vestibular system is responsible for detecting rotational and translational movements of the head and its orientation with respect to gravity, thereby controlling gaze stability, postural control, and locomotion. Recent evidence has also demonstrated that vestibular signals are projected to many subcortical

\footnotetext{
${ }^{*}$ Corresponding author: Monirah Albathi, MBBS, Post Doctoral Research Fellow, Department of Otolaryngology-Head and Neck Surgery, Johns Hopkins University School of Medicine, 601 N, Caroline Street - 6th Floor JHOC, Baltimore, MD 212870910, USA. Tel.: +1 312619 7012; E-mail: malbath1@jhu.edu.
}

and cortical structures including the insula, parietal operculum, temporoparietal junction, somatosensory cortex, frontal eye fields, hippocampus, cingulate cortex, and intraparietal sulcus (Lopez and Blanke 2011, Dieterich and Brandt 2015, Lopez C 2015). Vestibular influences on processes beyond balance and gait are increasingly being recognized.

Multiple reports have examined the relationship between vestibular function and sleep. Several mechanisms have been proposed to explain the link between the vestibular system and sleep. The vestibular system carries information about the positional location of the head, and may thus play a role in 
sleep initiation and duration (SI Wiener 2005). Additionally, results from animal studies suggest that the vestibular system is involved in regulating circadian rhythms by working in tandem with input from the visual and somatosensory systems (Fuller and Fuller 2006, Martin, Mauvieux et al. 2015). Further, a study of 9 adult patients found that patients with bilateral vestibular loss had abnormal sleep patterns and shorter sleep duration relative to healthy controls (Martin, Moussay et al. 2016). Whether the relationship between vestibular function and sleep holds in a large population-based cohort has not been investigated.

In this report, we use data from the 2008 National Health Interview Survey (NHIS) to examine the association between vestibular function and sleep. We used a well-validated definition of vestibular loss that has been previously applied to the NHIS data (Neuhauser, von Brevern et al. 2005, Bigelow, Semenov et al. 2015). With respect to sleep, the NHIS specifically queried participants about sleep duration, which we used in our analyses. We examined the independent relationship between sleep duration and vestibular vertigo with the hypothesis that those suffering from vestibular vertigo would report aberrant sleep durations (Parsons, Moriarity et al. 2014).

\section{Methods}

The sample for this cross-sectional study was extracted from the 2008 NHIS, which contained a supplement for dizziness and balance problems (Parsons, Moriarity et al. 2014). In 2008, the National Institute on Deafness and Other Communication Disorders sponsored the first nationally representative survey for balance and hearing disorders, which included a broad range of questions on balance and dizziness problems. More recent iterations of the NHIS (2009-2015) do not include questions on balance problems, and therefore could not be used for investigating the relationship between vestibular vertigo and sleep. The supplemental survey began by asking if the person had experienced balance or dizziness problems within the past 12 months, and those who answered 'yes' were then asked a series of follow-up questions. The NHIS is a multistage area probability design household survey used by the United States National Center for Health Statistics to monitor health status. These data are publically available through the NHIS website, and details on sampling methods and survey design can be found in the "NHIS Survey Description" (Pleis, Lucas et al. 2009). The US Census Bureau conducts these interviews using stratification, multistage sampling, and a probability cluster sampling technique with oversampling of subpopulations to produce generalizable estimates of the civilian, non-institutionalized U.S. population. The study was conducted in accordance with the Declaration of Helsinki, and IRB approval was not obtained because these data are de-identified and publically available.

\subsection{Defining vestibular vertigo}

We generated a single vestibular vertigo variable based on a definition first developed and validated by Neuhauser and colleagues in the German population (Neuhauser, von Brevern et al. 2005), and subsequently updated and used by several authors to define vestibular vertigo within the NHIS survey (Ward, Agrawal et al. 2013, Bigelow, Semenov et al. 2015). Figure 1 is a flowchart illustrating the development of the case definition variable for vestibular vertigo. Participants, who answered 'yes' to the first question, were then asked a series of questions about the nature of their problem. Participants with a history of stroke, movement disorders, multiple sclerosis, spine injury, muscular dystrophy, glaucoma, diabetic retinopathy, cataracts, and macular degeneration were not included in the vestibular vertigo case definition.

\subsection{Defining sleep duration}

In the NHIS data, sleep duration was recorded in hour increments. The sleep duration variable was available for all adults 18 years or older in the 2008 survey. Previous studies have categorized sleep duration as short (less than 6 hours), normal (6 to 8 hours), or long (more than 8 hours) per 24-hour period (Nunes, Jean-Louis et al. 2008, Ramos, Wallace et al. 2014, Akinseye, Ojike et al. 2016). Sleeping less than 6 hours or more than 8 hours, has been associated with various adverse health outcomes (Ayas, White et al. 2003, Grandner and Kripke 2004, Grandner, Hale et al. 2010, Akinseye, Ojike et al. 2016). Therefore, we used this same classification for sleep duration in the current study.

\subsection{Identifying covariates and possible confounders}

Vestibular vertigo has been reported to have an increased prevalence in females, (Neuhauser, 
Vestibular Vertigo Case Definition

Based on NHIS Survey

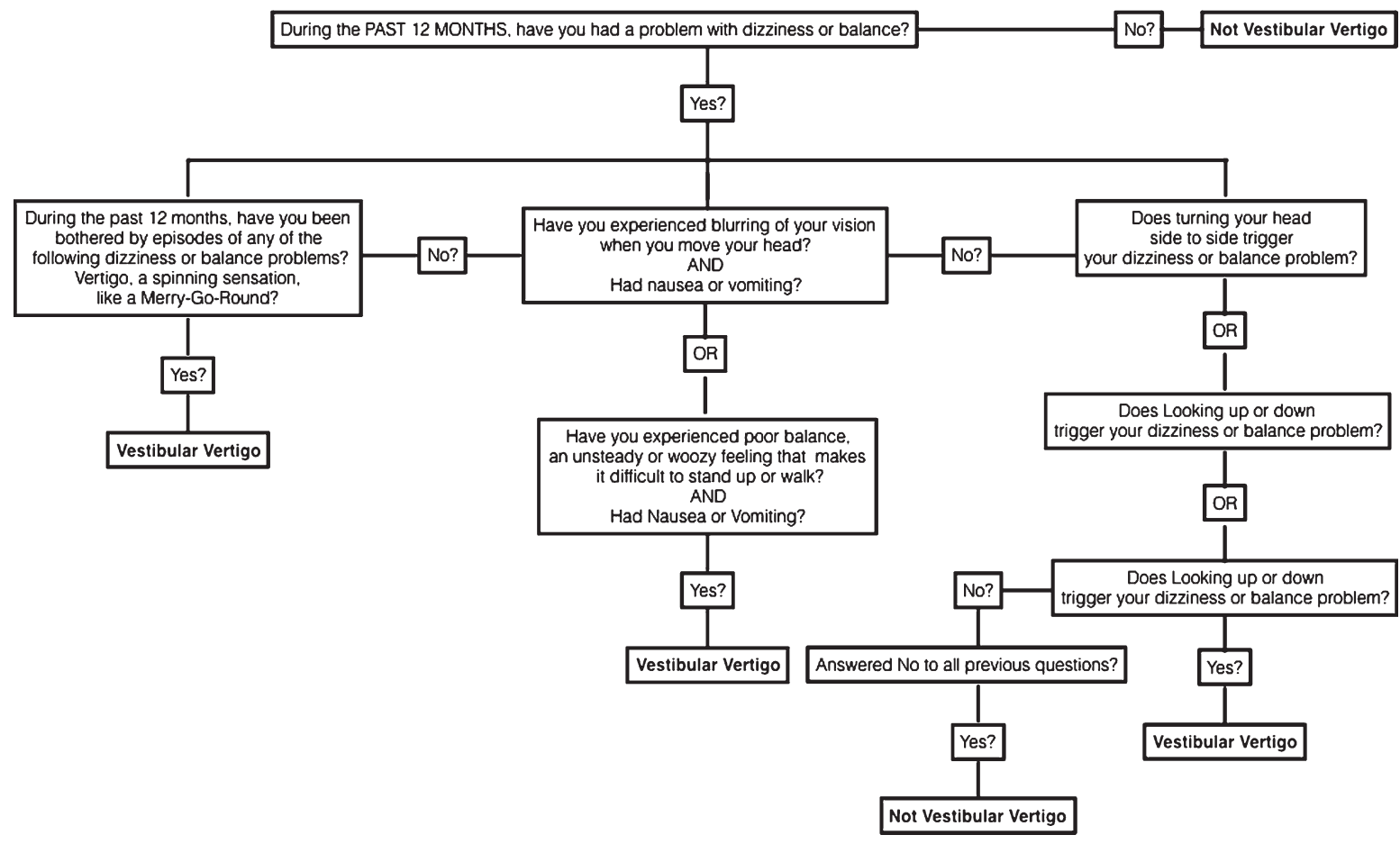

Fig. 1. A flowchart illustrating the case definition for vestibular vertigo from the 2008 National Health Interview Survey. Patients were excluded from the vestibular vertigo definition if they history of any of the following: stroke, movement disorder, multiple sclerosis, spine injury, muscular dystrophy, macular degeneration, glaucoma, diabetic retinopathy, or cataract.

von Brevern et al. 2005, Neuhauser 2007, Bigelow, Semenov et al. 2015) older adults, (Neuhauser, von Brevern et al. 2005, Neuhauser 2007, Ward, Agrawal et al. 2013, Bigelow, Semenov et al. 2015) individuals at lower socioeconomic status, (Agrawal, Carey et al. 2009) diabetics, (Agrawal, Carey et al. 2009, Agrawal, Carey et al. 2010, Buxton and Marcelli 2010) and individuals with psychiatric or cognitive impairment (Bigelow, Semenov et al. 2015). Disturbed sleep patterns have been reported in older individuals, (Pollak, Perlick et al. 1990, Prinz, Vitiello et al. 1990, Bliwise, King et al. 1992, Bliwise 1993) African-Americans, (Nunes, Jean-Louis et al. 2008, Brimah, Oulds et al. 2013) and individuals with diabetes, (Akinseye, Ojike et al. 2016) cardiovascular disease, (Partinen, Putkonen et al. 1982, Qureshi, Giles et al. 1997, Sabanayagam and Shankar 2010, Sabanayagam and Shankar 2012, Akinseye, Ojike et al. 2016) obesity, (Hasler, Buysse et al. 2004, Cizza, Skarulis et al. 2005, Jean-Louis, Williams et al. 2014) visual impairment, (Ramos, Wallace et al. 2014) and psychiatric illness (Bliwise, Friedman et al. 1993).
We accounted for these potentially confounding variables in our analyses. Age was categorized into three groups: $18-39$ years, $40-59$ years, and 60 years or older. Race was classified into five categories: nonHispanic white, non-Hispanic Black, Asian, Hispanic and other or mixed-race. Education level was classified into three categories: less than high school, graduated high school or has GED, and has an additional degree after high school. BMI was classified into 4 categories based on Centers for Disease Control and Prevention recommended cut-offs (Barlow and Dietz 1998). Details on how the variables for smoking, hypertension, diabetes, asthma, depression, generalized anxiety, panic disorder and chronic fatigue were collected for the NHIS have been previously described (Pleis, Lucas et al. 2009, Bigelow, Semenov et al. 2015).

\subsection{Statistical analysis}

Analyses were performed on the sample data, and then weighted to produce US population estimates 
and prevalence estimates as per NHIS survey sampling design guidelines (Pleis, Lucas et al. 2009). Data that were not within the sampling unit for a specified variable were coded as missing. Prevalence estimates were generated for the overall population and the subpopulation with vestibular vertigo. Simple logistic regressions were used to estimate the crude odds ratio $(\mathrm{OR})$ of each sleep duration category with respect to the others associated with having vestibular vertigo. Multinomial logistic regressions were used to compare the relative risk ratio (RRR) of short and long sleep relative to normal sleep duration associated with vestibular vertigo. Crude (only sleep duration and vestibular vertigo, with normal sleep duration as the baseline) and adjusted (for all covariates) multinomial regression analyses were performed to examine confounders. We checked the fit of our final model using likelihood ratio tests. STATA V.14 was used to perform all analyses (Jones, Jampani et al. 2000).

\section{Results}

In 2008, 20,950 individuals completed the balance and dizziness supplement. Conditional adult sample response rate for the 2008 survey was $74.2 \%$. Mean age for this population was $45.4 \pm 17.3$ years and ranged from $18-85$ years. Sex distribution was $52.6 \%$ female and $47.5 \%$ male. Race distribution was $56.4 \%$ White, 14.5\% African American, 20.3\% Hispanic, and $9.10 \%$ other races. The prevalence of normal sleep duration was lower in the vestibular vertigo population (69.7\% versus $82.2 \%$ ), while the prevalence of short and long sleep was higher $(15.5 \%$ versus $7.90 \%$ for short sleep, and $14.8 \%$ versus $8.80 \%$ for

Table 1

US Population Prevalence Estimates of Demographic and Comorbid Conditions from 2008 NHIS Data

\begin{tabular}{|c|c|c|c|c|}
\hline \multirow[b]{2}{*}{ Variable Prevalence within US Adult Population } & \multicolumn{2}{|c|}{ All Respondents } & \multicolumn{2}{|c|}{ Vestibular Vertigo } \\
\hline & Per Cent & $\begin{array}{c}216 \text { million } \\
95 \% \text { CI }\end{array}$ & Per Cent & $\begin{array}{c}18 \text { million } \\
95 \% \text { CI }\end{array}$ \\
\hline \multicolumn{5}{|l|}{ Sleep Duration } \\
\hline Short (Less than 6 hours) & $7.93 \%$ & {$[7.51-8.39]$} & $15.5 \%$ & {$[13.7-17.5]$} \\
\hline Normal (6-8 hours) & $83.2 \%$ & {$[82.6-83.8]$} & $69.7 \%$ & {$[66.9-72.3]$} \\
\hline Long (More than 8 hours) & $8.83 \%$ & {$[8.38-9.31]$} & $14.78 \%$ & [12.9-16.9] \\
\hline \multicolumn{5}{|l|}{ Age } \\
\hline $18-39$ years & $39.6 \%$ & {$[38.6-40.6]$} & $30.2 \%$ & {$[27.4-33.2]$} \\
\hline $40-59$ years & $35.5 \%$ & {$[34.7-36.3]$} & $37.9 \%$ & {$[35.0-40.8]$} \\
\hline 60 or older & $24.9 \%$ & {$[24.1-25.7]$} & $31.9 \%$ & [29.6-34.4] \\
\hline \multicolumn{5}{|l|}{ Sex } \\
\hline Male & $49.0 \%$ & {$[48.3-49.7]$} & $34.0 \%$ & [31.3-36.7] \\
\hline Female & $51.0 \%$ & {$[50.3-51.7]$} & $66.0 \%$ & {$[63.3-68.7]$} \\
\hline \multicolumn{5}{|l|}{ Race } \\
\hline White & $68.7 \%$ & [67.7-69.7] & $72.8 \%$ & {$[70.3-75.2]$} \\
\hline Black & $11.6 \%$ & [10.9-12.4] & $9.16 \%$ & [7.86-10.7] \\
\hline Asian & $4.51 \%$ & {$[4.19-4.85]$} & $2.3 \%$ & {$[1.61-3.30]$} \\
\hline Hispanic & $13.0 \%$ & {$[12.3-13.8]$} & $12.3 \%$ & {$[10.5-14.4]$} \\
\hline Other & $2.21 \%$ & {$[1.90-2.57]$} & $3.39 \%$ & {$[2.49-4.62]$} \\
\hline \multicolumn{5}{|l|}{ Education Level } \\
\hline Less than High School & $15.5 \%$ & {$[14.7-16.2]$} & $19.5 \%$ & {$[17.4-21.8]$} \\
\hline High School or GED & $27.7 \%$ & [26.9-28.6] & $29.0 \%$ & {$[26.4-31.8]$} \\
\hline More than High School & $56.9 \%$ & [55.9-57.9] & $51.5 \%$ & {$[48.8-54.2]$} \\
\hline \multicolumn{5}{|l|}{ Body Mass Index } \\
\hline Underweight & $1.57 \%$ & {$[1.38-1.80]$} & $1.64 \%$ & {$[1.10-2.44]$} \\
\hline Normal & $34.3 \%$ & {$[33.5-35.1]$} & $30.5 \%$ & {$[28.0-33.2]$} \\
\hline Overweight & $33.3 \%$ & [32.6-34.0] & $29.3 \%$ & {$[27.0-31.7]$} \\
\hline Obese & $30.9 \%$ & {$[30.1-31.7]$} & $38.6 \%$ & {$[35.8-41.5]$} \\
\hline Ever Smoker & $42.2 \%$ & [41.3-43.5] & $53.31 \%$ & {$[50.7-55.9]$} \\
\hline Diabetes Mellitus & $8.28 \%$ & {$[7.84-8.75]$} & $15.2 \%$ & [13.3-17.3] \\
\hline Hypertension & $29.3 \%$ & [28.7-30.3] & $43.6 \%$ & {$[40.8-46.5]$} \\
\hline Major Depression Disorder & $28.2 \%$ & {$[27.4-29.1]$} & $61.6 \%$ & {$[58.8-64.3]$} \\
\hline Panic Disorder & $7.99 \%$ & {$[7.55-8.44]$} & $25.8 \%$ & [23.6-28.1] \\
\hline Generalized Anxiety Disorder & $19.3 \%$ & [18.6-20.0] & $46.2 \%$ & [43.4-48.9] \\
\hline Chronic Fatigue & $2.31 \%$ & {$[2.11-2.54]$} & $10.6 \%$ & {$[9.10-12.2]$} \\
\hline Asthma & $12.6 \%$ & {$[12.0-13.1]$} & $21.8 \%$ & [19.7-24.1] \\
\hline
\end{tabular}

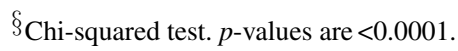


Table 2

Multinomial logistic regression models of sleep duration categories, and vestibular vertigo, from the 2008 National Health Interview Survey

\begin{tabular}{lccc}
\hline Sleep Duration & $\begin{array}{c}\text { Simple Logistic } \\
\text { Regression }\end{array}$ & $\begin{array}{c}\text { Multinomial Logistic Regression } \\
\text { Unadjusted }\end{array}$ & $\begin{array}{c}\text { Multinomial Logistic Regression }^{\mathrm{b}} \\
\text { Adjusted }^{\mathrm{b}}\end{array}$ \\
\hline Short (Less than 6 hours) & $2.40[2.10-2.75]$ & $2.59[2.19-3.06]$ & $1.75[1.45-2.11]$ \\
& $(p<0.001)$ & $(p<0.001)$ & $(p<0.001)$ \\
Normal (6-8 hours) & $0.45[0.40-0.50]$ & 1 & 1 \\
& $(p<0.001)$ & Base Outcome & Base Outcome \\
Long (More than 8 hours) & $1.68[1.47-1.93]$ & $2.17[1.81-2.59]$ & $1.55[1.26-1.91]$ \\
& $(p<0.001)$ & $(p<0.001)$ & $(p<0.001)$ \\
\hline
\end{tabular}

${ }^{\text {a }}$ Population size is approximately 216 million for these estimates. ${ }^{b}$ Population size is approximately 200 million for these estimates. ${ }^{c}$ For age, race, education level, income, BMI, smoking history, diabetes, hypertension, asthma, chronic fatigue, depression, panic disorder and generalized anxiety disorder.

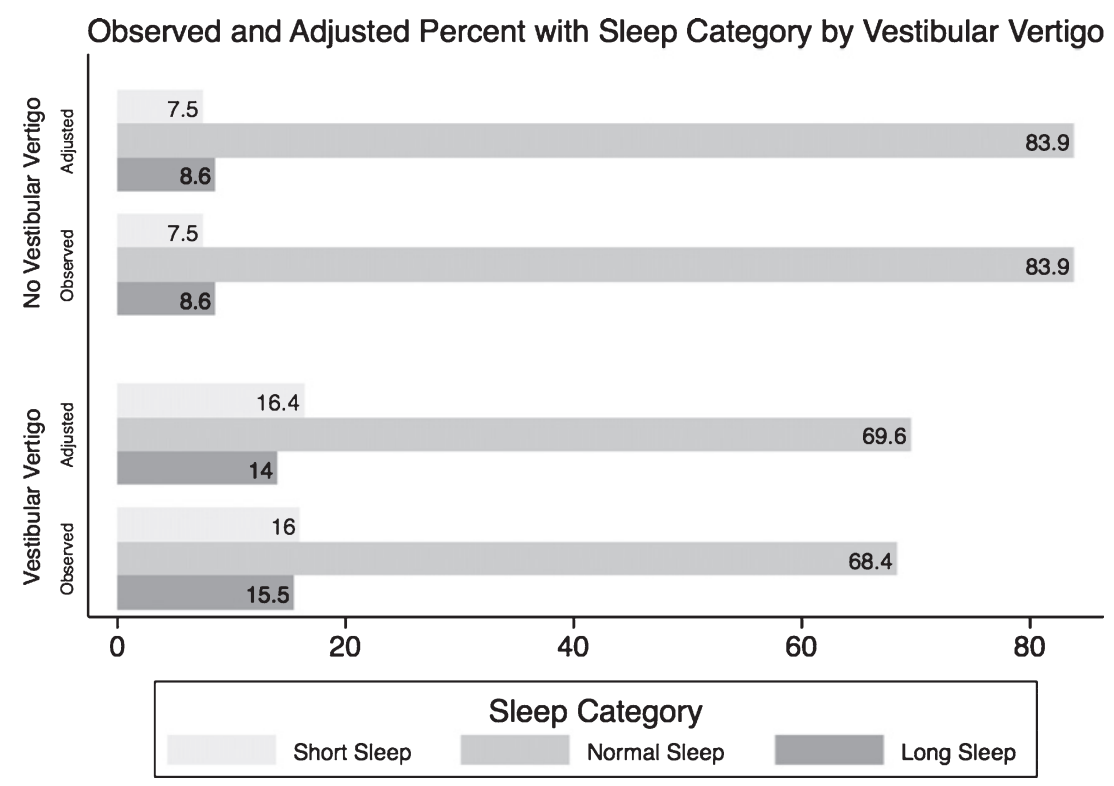

Fig. 2. A graph comparing the observed (crude) and the predicted (adjusted) percentages of each sleep category in normal individuals and in people suffering from vestibular vertigo. *From the Null Multinomial Logistic Regression of Sleep Categories and Vestibular Vertigo only. ^From Extended Multinomial Logistic Regression model including the following covariates: age, race, education level, income, BMI, smoking history, diabetes, hypertension, asthma, chronic fatigue, depression, panic disorder and generalized anxiety disorder.

long sleep). In addition, all health conditions listed in Table 1 have a higher prevalence in the vestibular population compared to the overall US population. Table 1 summarizes findings from 2008 NHIS data on the demographic and associated comorbid conditions of the US population and their prevalence with vestibular vertigo.

Individuals with vestibular vertigo have an adjusted RRR of 1.75 (95\% CI 1.45-2.11; pvalue $<0.001$ ) of sleeping less than 6 hours a day relative to regular sleep duration, and an adjusted RRR of 1.55 (95\% CI 1.26-1.91; $p$-value $<0.001$ ) of sleeping more than 8 hours a day relative to regular sleep duration, compared to individuals without vestibular vertigo. Table 2 summarizes the unadjusted, and the subsequent adjusted regressions for the ORs and RRRs of the three sleep categories associated with having vestibular vertigo. Figure 2 shows the observed percentages that were presented in Table 1 of each sleep category in individuals with and without vestibular vertigo, and the subsequent adjusted proportion of each sleep category after accounting for all covariates using the multinomial regression analysis.

\section{Discussion}

Our study estimates that individuals with vestibular vertigo had a 1.75 RRR of having short sleep duration compared to individuals without vestibular vertigo and a $1.55 \mathrm{RRR}$ of having long sleep duration 
compared to individuals without vestibular vertigo. The multinomial logistic regression model used in our study allowed us to compare the RRR's of short sleep and long sleep relative to normal sleep associated with vestibular vertigo. We observed a greater RRR for short sleep relative to long sleep duration associated with vestibular vertigo; that is, the relationship between vestibular vertigo and sleep duration was stronger for short sleep duration. After adjusting for variables known to be associated with abnormal selfreported sleep duration, as demonstrated in Fig. 2, individuals with vestibular vertigo reported an $8 \%$ higher prevalence of shorter sleep duration than individuals without vestibular vertigo, and a $5 \%$ higher prevalence of longer sleep duration.

The effect of abnormal sleep duration on overall health is an evolving area of research. The mechanisms behind abnormally short or long sleep duration are not fully understood. Abnormal sleep duration has been associated with a number of adverse cardiovascular, endocrine, neurologic and psychiatric conditions as well as increased mortality (Bliwise, Friedman et al. 1993, Ayas, White et al. 2003, Grandner and Kripke 2004, Youngstedt and Kripke 2004, Buxton and Marcelli 2010, Sabanayagam and Shankar 2010). A meta-analysis of over 60 studies on sleep duration observed a U-shaped relationship between sleep duration and mortality, whereby mortality risk increases with further deviation from the normal sleep range (Grandner, Hale et al. 2010). Short sleep, in particular, appears to pose a greater risk of morbidity and mortality (Brassington, King et al. 2000). In animal models, short sleep was associated with weight loss, increased energy expenditure, increased plasma norepinephrine, and decreased plasma thyroxine, eventually culminating in death (Rechtschaffen and Bergmann 2002, Rechtschaffen, Bergmann et al. 2002). Short sleep has also been linked to increased falls in older adults (Brassington, King et al. 2000). The link between short sleep and vestibular vertigo may in part account for this association. Long sleep, on the other hand, has been associated with sleep fragmentation, fatigue, and changes in immune function, which also increase morbidity and mortality (Grandner and Drummond 2007).

Several potential mechanisms may explain the association between vestibular vertigo and altered sleep duration. The vestibular system, which senses head position with respect to gravity, may provide important cues for sleep initiation and maintenance. Experiments carried out in simulated hypergravity conditions on knockout mice (het -/-) selectively devoid of otolithic function first introduced the hypothesis that vestibular neuronal input represented by macular signaling activates nuclei throughout the vestibulo-cortical neuraxis including autonomic, limbic and hypothalamic nuclei (Fuller, Jones et al. 2004). Findings from a more recent study by Besnard et al. suggest that vestibular input directly influences the suprachiasmatic nucleus, thereby having a potential regulatory effect on circadian rhythmicity in humans (Martin, Mauvieux et al. 2015).

However, the directionality of the association is an area of evolving research because other studies suggest that abnormal sleep duration may impact vestibular signaling. One study found that sleep deprivation has been shown to alter cortical function within the posterior parietal cortex, which may in turn alter vestibular processing of information, (Drummond, Gillin et al. 2001) while Martin and colleagues reported in their study of adult patients with bilateral vestibular dysfunction that these patients had significantly elevated levels of salivary cortisol compared to healthy controls (Martin, Moussay et al. 2016). In theory, the elevation in cortisol could be attributed to both the stress of disturbed sleep patterns and to vestibular dysfunction, and it has been shown that chronically elevated levels of cortisol may contribute to hippocampal atrophy. This last point was elaborated further in other studies that found correlations between hippocampal atrophy and bilateral vestibular loss (Van Cruijsen, Hiemstra et al. 2007, Aitken, Benoit et al. 2016, Balabhadrapatruni, Zheng et al. 2016, Seo, Kim et al. 2016).

Another hypothesis that could explain the link between sleep duration and vestibular loss are the known vestibular inputs to the autonomic nervous system (Yates 1996, Yates and Bronstein 2005). The vestibular system has been shown to control autonomic functions during rapid eye movement sleep (Morrison and Pompeiano 1970). Krystal et al. reported that electrical stimulation of the vestibular apparatus decreased sleep latency in individuals with sleep latency $>14$ minutes (Krystal, Zammit et al. 2010). Lastly, in a recent study, Lin and Young found that sleep-deprivation of more than 12 hours resulted in increased asymmetry of oVEMPs, although had no effect on cVEMPs (Lin and Young 2014).

\section{Limitations}

Our results may be confounded by other possible causes of sleep disturbance that were not available as 
part of this historic dataset. We were limited by the variables that were collected during the 2008 NHIS interview, and therefore some confounding variables may have been missed. Furthermore, the directionality of this relationship cannot be ascertained based on cross-sectional data. We are therefore unable to rule out the possibility of reverse causality, wherein sleep disturbance causes vestibular vertigo, and not the other way around. Prospective, longitudinal studies that clearly stratify patients based on established risks for sleep disturbance and vestibular disease are needed to better explain the influence of these two variables on each other.

Given that we performed a cross-sectional analysis looking at a dependent variable that could be affected by many covariates, over-adjustment and under-adjustment are concerns. The importance of under-adjustment was previously underscored by Patel et al. who have demonstrated a $22 \%$ decrease in their crude mortality risk after accounting for a variety of possible confounders (Patel, Ayas et al. 2004). On the other hand, with 14 covariates in our final model, we run the risk of over adjusting for variables that may actually be on the causal pathway between vestibular vertigo and sleep, particularly depression, chronic fatigue, and obesity. We tried to overcome this by running several sensitivity analyses, but the independent effect of individual covariates on sleep remains difficult to discern from cross-sectional data. The 2008 NHIS only included one question about sleep that described sleep duration. Sleep quality, encompassing sleep onset latency, sleep interruption, and early awakening, and the effect of sleep quality on daily life were not captured in these data. We therefore are basing our results on one facet of sleep assessment. Future prospective studies should incorporate validated surveys that capture information about sleep quality, such as the Pittsburgh Sleep Quality Index, (Buysse, Reynolds et al. 1989) and impact of poor sleep on activities of daily living, such as the Epworth Sleepiness Scale (Johns 1991) in order to gain true insight into the effect of sleep disturbance on the quality of life of patients with vestibular vertigo. Additionally, the NHIS does not directly capture comorbid sleep conditions such as insomnia, circadian rhythm disorders, and sleep-related breathing disorders. Most notably, obstructive sleep apnea, a common cause of sleep disturbance, is not directly accounted for in this dataset and is therefore approximated using body mass index estimates as a covariate. Future studies investigating the relationship between vestibular dysfunction and sleep should account for these disorders in their data collection and analyses.

Finally, studies examining reliability of subjective sleep duration measures, such as self-reported sleep, reported conflicting results about their agreement with more objective measures such as actigraphy and polysomnography (Akerstedt, Hume et al. 1994, Lockley, Skene et al. 1999, Landis, Frey et al. 2003, Girschik, Fritschi et al. 2012). The majority of studies report generally poor agreement between the subjective questions used in surveys and objective sleep testing, and recommend integrating both objective and subjective measures whenever possible (Argyropoulos, Hicks et al. 2003, Van Den Berg, Van Rooij et al. 2008, van den Berg, Miedema et al. 2009, Segura-Jimenez, Camiletti-Moiron et al. 2015). It is also important to note that short sleep does not necessarily translate into sleep insufficiency, and long sleep may be over-reported to encompass both time spent sleeping and time spent in bed trying to sleep. Responses may be biased towards the null because self-reported sleep may contain considerable error and individuals may misreport how long they actually sleep to durations that they perceive to be more desirable (Coates, Killen et al. 1982, Bliwise, King et al. 1992, Bliwise, Friedman et al. 1993). This is why future studies should aim to utilize data from both subjective and objective sleep assessment tools, and correlate their results.

\section{Conclusions}

This study presents epidemiologic evidence obtained through robust statistical analyses for an association between vestibular function and sleep duration. Patients with vestibular vertigo have a higher RRR for disturbed sleep patterns, which may contribute to worsening quality of life and may predispose this patient population to other adverse health sequelae. While the mechanisms behind the link between vestibular vertigo and abnormal sleep require further study, increased recognition of sleep impairments among individuals with vestibular vertigo might be useful in the clinical setting.

\section{Acknowledgments}

The authors would like to thank Dr. Robin Bigelow for his contribution in preparing the data for analysis. 


\section{References}

Y. Agrawal, J.P. Carey, C.C. Della Santina, M.C. Schubert and L.B. Minor, Disorders of balance and vestibular function in US adults: Data from the National Health and Nutrition Examination Survey, 2001-2004, Arch Intern Med 169(10) (2009), 938-944.

Y. Agrawal, J.P. Carey, C.C. Della Santina, M.C. Schubert and L.B. Minor, Diabetes, vestibular dysfunction, and falls: Analyses from the National Health and Nutrition Examination Survey, Otol Neurotol 31(9) (2010), 1445-1450.

P. Aitken, A. Benoit, Y. Zheng, B. Philoxene, A. Le Gall, P. Denise, S. Besnard and P.F. Smith, Hippocampal and striatal M1 -muscarinic acetylcholine receptors are down-regulated following bilateral vestibular loss in rats, Hippocampus 26(12) (2016), 1509-1514.

T. Akerstedt, K. Hume, D. Minors and J. Waterhouse, The meaning of good sleep: A longitudinal study of polysomnography and subjective sleep quality, J Sleep Res 3(3) (1994), 152-158.

O.A. Akinseye, N.I. Ojike, L.I. Akinseye, P.S. Dhandapany and S.R. Pandi-Perumal, Association of Sleep Duration with Stroke in Diabetic Patients: Analysis of the National Health Interview Survey, J Stroke Cerebrovasc Dis 25(3) (2016), 650-655.

S.V. Argyropoulos, J.A. Hicks, J.R. Nash, C.J. Bell, A.S. Rich, D.J. Nutt and S.J. Wilson, Correlation of subjective and objective sleep measurements at different stages of the treatment of depression, Psychiatry Res 120(2) (2003), 179-190.

N.T. Ayas, D.P. White, W.K. Al-Delaimy, J.E. Manson, M.J. Stampfer, F.E. Speizer, S. Patel and F.B. Hu, A prospective study of self-reported sleep duration and incident diabetes in women, Diabetes Care 26(2) (2003), 380-384.

S. Balabhadrapatruni, Y. Zheng, R. Napper and P.F. Smith, Basal dendritic length is reduced in the rat hippocampus following bilateral vestibular deafferentation, Neurobiol Learn Mem 131 (2016), 56-60.

S.E. Barlow and W.H. Dietz, Obesity evaluation and treatment: Expert Committee recommendations. The Maternal and Child Health Bureau, Health Resources and Services Administration and the Department of Health and Human Services, Pediatrics 102(3) (1998), E29.

R.T. Bigelow, Y.R. Semenov, S. du Lac, H.J. Hoffman and Y. Agrawal, Vestibular vertigo and comorbid cognitive and psychiatric impairment: The 2008 National Health Interview Survey, J Neurol Neurosurg Psychiatry 2015.

D.L. Bliwise, Sleep in normal aging and dementia, Sleep 16(1) (1993), 40-81.

D.L. Bliwise, L. Friedman and J.A. Yesavage, Depression as a confounding variable in the estimation of habitual sleep time, J Clin Psychol 49(4) (1993), 471-477.

D.L. Bliwise, A.C. King, R.B. Harris and W.L. Haskell, Prevalence of self-reported poor sleep in a healthy population aged 50-65, Soc Sci Med 34(1) (1992), 49-55.

G.S. Brassington, A.C. King and D.L. Bliwise, Sleep problems as a risk factor for falls in a sample of community-dwelling adults aged 64-99 years, J Am Geriatr Soc 48(10) (2000), 1234-1240.

P. Brimah, F. Oulds, O. Olafiranye, M. Ceide, S. Dillon, O. Awoniyi, J. Nunes and G. Jean-Louis, Sleep duration and reported functional capacity among black and white US adults, J Clin Sleep Med 9(6) (2013), 605-609.

O.M. Buxton and E. Marcelli, Short and long sleep are positively associated with obesity, diabetes, hypertension, and cardiovas- cular disease among adults in the United States, Soc Sci Med 71(5) (2010), 1027-1036.

D.J. Buysse, C.F. Reynolds, 3rd, T.H. Monk, S.R. Berman and D.J. Kupfer, The Pittsburgh Sleep Quality Index: A new instrument for psychiatric practice and research, Psychiatry Res 28(2) (1989), 193-213.

G. Cizza, M. Skarulis and E. Mignot, A link between short sleep and obesity: Building the evidence for causation, Sleep 28(10) (2005), 1217-1220.

T.J. Coates, J.D. Killen, J. George, E. Marchini, S. Silverman and C. Thoresen, Estimating sleep parameters: A multitraitmultimethod analysis, J Consult Clin Psychol 50(3) (1982), 345-352.

M. Dieterich and T. Brandt, The bilateral central vestibular system: Its pathways, functions, and disorders, Ann N Y Acad Sci 1343 (2015), 10-26.

S.P. Drummond, J.C. Gillin and G.G. Brown, Increased cerebral response during a divided attention task following sleep deprivation, J Sleep Res 10(2) (2001), 85-92.

P.M. Fuller and C.A. Fuller, Genetic evidence for a neurovestibular influence on the mammalian circadian pacemaker, $J$ Biol Rhythms 21(3) (2006), 177-184.

P.M. Fuller, T.A. Jones, S.M. Jones and C.A. Fuller, Evidence for macular gravity receptor modulation of hypothalamic, limbic and autonomic nuclei, Neuroscience 129(2) (2004), 461-471.

J. Girschik, L. Fritschi, J. Heyworth and F. Waters, Validation of self-reported sleep against actigraphy, J Epidemiol 22(5) (2012), 462-468.

M.A. Grandner and S.P. Drummond, Who are the long sleepers? Towards an understanding of the mortality relationship, Sleep Med Rev 11(5) (2007), 341-360.

M.A. Grandner, L. Hale, M. Moore and N.P. Patel, Mortality associated with short sleep duration: The evidence, the possible mechanisms, and the future, Sleep Med Rev 14(3) (2010), 191-203.

M.A. Grandner and D.F. Kripke, Self-reported sleep complaints with long and short sleep: A nationally representative sample, Psychosom Med 66(2) (2004), 239-241.

G. Hasler, D.J. Buysse, R. Klaghofer, A. Gamma, V. Ajdacic, D. Eich, W. Rossler and J. Angst, The association between short sleep duration and obesity in young adults: A 13-year prospective study, Sleep 27(4) (2004), 661-666.

G. Jean-Louis, N.J. Williams, D. Sarpong, A. Pandey, S. Youngstedt, F. Zizi and G. Ogedegbe, Associations between inadequate sleep and obesity in the US adult population: Analysis of the national health interview survey, (1977-2009), BMC Public Health 14 (2014), 290.

M.W. Johns, A new method for measuring daytime sleepiness: The Epworth sleepiness scale, Sleep 14(6) (1991), 540-545.

R.D. Jones, H.B. Jampani, J.L. Newman and A.S. Lee, Triclosan: A review of effectiveness and safety in health care settings, $\mathrm{Am}$ J Infect Control 28(2) (2000), 184-196.

A.D. Krystal, G.K. Zammit, J.K. Wyatt, S.F. Quan, J.D. Edinger, D.P. White, R.P. Chiacchierini and A. Malhotra, The effect of vestibular stimulation in a four-hour sleep phase advance model of transient insomnia, J Clin Sleep Med 6(4) (2010), 315-321.

C.A. Landis, C.A. Frey, M.J. Lentz, J. Rothermel, D. Buchwald and J.L. Shaver, Self-reported sleep quality and fatigue correlates with actigraphy in midlife women with fibromyalgia, Nurs Res 52(3) (2003), 140-147. 
B.Y. Lin and Y.H. Young, Effect of short-duration sleep deprivation on the vestibulo-ocular reflex system evaluated by ocular vestibular-evoked myogenic potential test, Acta Otolaryngol 134(7) (2014), 698-703.

S.W. Lockley, D.J. Skene and J. Arendt, Comparison between subjective and actigraphic measurement of sleep and sleep rhythms, J Sleep Res 8(3) (1999), 175-183.

C. Lopez and O. Blanke, The thalamocortical vestibular system in animals and humans, Brain Res Rev 67(1-2) (2011), $119-146$

T.A. Lopez C, Vestibular Cortex. Brain mapping: An encyclopedic reference., Elsevier: Academic Press, (2015), 305-312.

T. Martin, B. Mauvieux, J. Bulla, G. Quarck, D. Davenne, P. Denise, B. Philoxene and S. Besnard, Vestibular loss disrupts daily rhythm in rats, J Appl Physiol (1985) 118(3) (2015), 310-318.

T. Martin, S. Moussay, I. Bulla, J. Bulla, M. Toupet, O. Etard, P. Denise, D. Davenne, A. Coquerel and G. Quarck, Exploration of Circadian Rhythms in Patients with Bilateral Vestibular Loss, PLoS One 11(6) (2016), e0155067.

A.R. Morrison and O. Pompeiano, Vestibular influences during sleep. VI. Vestibular control of autonomic functions during the rapid eye movements of desynchronized sleep, Arch Ital Biol 108(1) (1970), 154-180.

H.K. Neuhauser, Epidemiology of vertigo, Curr Opin Neurol 20(1) (2007), 40-46.

H.K. Neuhauser, M. von Brevern, A. Radtke, F. Lezius, M. Feldmann, T. Ziese and T. Lempert, Epidemiology of vestibular vertigo: A neurotologic survey of the general population, $\mathrm{Neu}$ rology 65(6) (2005), 898-904.

J. Nunes, G. Jean-Louis, F. Zizi, G.J. Casimir, H. von Gizycki, C.D. Brown and S.I. McFarlane, Sleep duration among black and white Americans: Results of the National Health Interview Survey, J Natl Med Assoc 100(3) (2008), 317-322.

V.L. Parsons, C. Moriarity, K. Jonas, T.F. Moore, K.E. Davis and L. Tompkins, Design and estimation for the national health interview survey, 2006-2015, Vital Health Stat 2(165) (2014), $1-53$.

M. Partinen, P.T. Putkonen, J. Kaprio, M. Koskenvuo and I. Hilakivi, Sleep disorders in relation to coronary heart disease, Acta Med Scand Suppl 660 (1982), 69-83.

S.R. Patel, N.T. Ayas, M.R. Malhotra, D.P. White, E.S. Schernhammer, F.E. Speizer, M.J. Stampfer and F.B. Hu, A prospective study of sleep duration and mortality risk in women, Sleep 27(3) (2004), 440-444.

J.R. Pleis, J.W. Lucas and B.W. Ward, Summary health statistics for U.S. adults: National Health Interview Survey, 2008, Vital Health Stat 10(242) (2009), 1-157.

C.P. Pollak, D. Perlick, J.P. Linsner, J. Wenston and F. Hsieh, Sleep problems in the community elderly as predictors of death and nursing home placement, J Community Health 15(2) (1990), 123-135.

P.N. Prinz, M.V. Vitiello, M.A. Raskind and M.J. Thorpy, Geriatrics: Sleep disorders and aging, N Engl J Med 323(8) (1990), $520-526$.
A.I. Qureshi, W.H. Giles, J.B. Croft and D.L. Bliwise, Habitual sleep patterns and risk for stroke and coronary heart disease: A 10-year follow-up from NHANES I, Neurology 48(4) (1997), 904-911.

A.R. Ramos, D.M. Wallace, N.J. Williams, D.W. Spence, S.R. Pandi-Perumal, F. Zizi and G. Jean-Louis, Association between visual impairment and sleep duration: Analysis of the 2009 National Health Interview Survey, (NHIS), BMC Ophthalmol 14 (2014), 115.

A. Rechtschaffen and B.M. Bergmann, Sleep deprivation in the rat: An update of the 1989 paper, Sleep 25(1) (2002), 18-24.

A. Rechtschaffen, B.M. Bergmann, C.A. Everson, C.A. Kushida and M.A. Gilliland, Sleep deprivation in the rat: X. Integration and discussion of the findings. 1989, Sleep 25(1) (2002), 68-87.

C. Sabanayagam and A. Shankar, Sleep duration and cardiovascular disease: Results from the National Health Interview Survey, Sleep 33(8) (2010), 1037-1042.

C. Sabanayagam and A. Shankar, Sleep duration and hypercholesterolaemia: Results from the National Health Interview Survey 2008, Sleep Med 13(2) (2012), 145-150.

V. Segura-Jimenez, D. Camiletti-Moiron, D. Munguia-Izquierdo, I. C. Alvarez-Gallardo, J.R. Ruiz, F.B. Ortega and M. DelgadoFernandez, Agreement between self-reported sleep patterns and actigraphy in fibromyalgia and healthy women, Clin Exp Rheumatol 33(1 Suppl 88) (2015), S58-67.

Y.J. Seo, J. Kim and S.H. Kim, The change of hippocampal volume and its relevance with inner ear function in Meniere's disease patients, Auris Nasus Larynx 43(6) (2016), 620-625.

J.T. SI Wiener, Head Direction Cells and the Neural Mechanisms of Spatial Orientation. Cambridge, MA, MIT Press 2005.

N. Van Cruijsen, W.M. Hiemstra, L.C. Meiners, H.P. Wit and F.W. Albers, Hippocampal volume measurement in patients with Meniere's disease: A pilot study, Acta Otolaryngol 127(10) (2007), 1018-1023.

J.F. van den Berg, H.M. Miedema, J.H. Tulen, A. Hofman, A.K. Neven and H. Tiemeier, Sex differences in subjective and actigraphic sleep measures: A population-based study of elderly persons, Sleep 32(10) (2009), 1367-1375.

J.F. Van Den Berg, F.J. Van Rooij, H. Vos, J.H. Tulen, A. Hofman, H.M. Miedema, A.K. Neven and H. Tiemeier, Disagreement between subjective and actigraphic measures of sleep duration in a population-based study of elderly persons, J Sleep Res 17(3) (2008), 295-302.

B.K. Ward, Y. Agrawal, H.J. Hoffman, J.P. Carey and C.C. Della Santina, Prevalence and impact of bilateral vestibular hypofunction: Results from the 2008 US National Health Interview Survey, JAMA Otolaryngol Head Neck Surg 139(8) (2013), 803-810.

B.J. Yates, Vestibular influences on the autonomic nervous system, Ann N Y Acad Sci 781 (1996), 458-473.

B.J. Yates and A.M. Bronstein, The effects of vestibular system lesions on autonomic regulation: Observations, mechanisms, and clinical implications, J Vestib Res 15(3) (2005), 119-129.

S.D. Youngstedt and D.F. Kripke, Long sleep and mortality: Rationale for sleep restriction, Sleep Med Rev 8(3) (2004), 159-174. 